\title{
A Novel Method for Optimal Solution of Fuzzy Chance Constraint Single-Period Inventory Model
}

\author{
Anuradha Sahoo and J. K. Dash \\ Department of Mathematics, ITER, Siksha 'O' Anusandhan University, Bhubaneswar, Odisha 751030, India \\ Correspondence should be addressed to Anuradha Sahoo; anuradha25anu@gmail.com
}

Received 16 July 2016; Accepted 11 October 2016

Academic Editor: Erich Peter Klement

Copyright ( 2016 A. Sahoo and J. K. Dash. This is an open access article distributed under the Creative Commons Attribution License, which permits unrestricted use, distribution, and reproduction in any medium, provided the original work is properly cited.

A method is proposed for solving single-period inventory fuzzy probabilistic model (SPIFPM) with fuzzy demand and fuzzy storage space under a chance constraint. Our objective is to maximize the total profit for both overstock and understock situations, where the demand $\widetilde{D}_{j}$ for each product $j$ in the objective function is considered as a fuzzy random variable (FRV) and with the available storage space area $\widetilde{W}$, which is also a FRV under normal distribution and exponential distribution. Initially we used the weighted sum method to consider both overstock and understock situations. Then the fuzziness of the model is removed by ranking function method and the randomness of the model is removed by chance constrained programming problem, which is a deterministic nonlinear programming problem (NLPP) model. Finally this NLPP is solved by using LINGO software. To validate and to demonstrate the results of the proposed model, numerical examples are given.

\section{Introduction}

One of the special parts of inventory model is single-period inventory model (SPIM). The objective of this SPIM is to determine the optimal order quantity, where the order for each item is only at the beginning of each work period, that is, for a day, a week, a month, and so forth, to maximize the expected profit or to minimize the cost. The use of SPIM can be found in fashion products business, food business, sporting, style goods, newspapers, Christmas trees, and so forth. In real life situations to estimate the market demand is a main factor for maximizing the profit or for minimizing the cost. There are the cases in which the probability distribution of the demand for the new items is completely unknown due to lack of historical data and sometimes due to technical difficulties or economic reasons or using the linguistic expression by the expert for demand forecasting. This deals with situations where the demand of products is uncertain. For these demand uncertainties, fuzzy set theory has been applied to inventory models.

Initially Zadeh [1] introduced a feasible approach to deal with this kind of fuzzy problem and established a fuzzy set which is characterized by a membership function which assigns to each object a grade of membership ranging between zero and one. Later depending upon different requirements of measurability, it was developed and extended by several researchers like Buckley [2], Kwakernaak [3], and Zimmermann [4] et al. Many researchers have done a lot of work in this area.

Nanda and Kar [5] discussed the concept of convexity and logarithmic convexity for fuzzy mappings and some applications to optimization are introduced. Puri and Ralescu [6] defined the concepts of fuzzy random variable and the expectation of a fuzzy random variable.

Petrović et al. [7] presented two fuzzy models for the newsboy problem in an uncertain environment where the uncertainties appear in demand and in inventory costs. Multi-item stochastic and fuzzy-stochastic inventory models are formulated under total budgetary and space constraints where shortages are allowed but fully backlogged by Das et al. [8]. Nanda et al. [9] presented a chance constrained programming model, where both fuzziness and randomness are present in the objective function and constraints.

Mahapatra and Maiti [10] developed a multiobjective and single-objective inventory models of stochastically deteriorating items in which demand is a function of inventory 
level and selling price of the commodity. They derived the results for both without shortages and partially backlogged shortages. Also objectives for profit maximization for each item are separately formulated with different goals and compromise solutions of the multiobjective production inventory problems are obtained by goal programming method.

Chang et al. [11] considered the mixture inventory model involving variable lead time with back orders and lost sales. They used the centroid method of defuzzification to estimate total cost in the fuzzy sense and found the optimal solution for order quantity and lead time in the fuzzy sense such that the total cost has a minimum value.

Shao and Ji [12] considered the multiproduct newsboy problem with fuzzy demands under budget constraint. They developed three types of models under different criteria in which the objective functions are to maximize the expected profit of newsboy, the chance of achieving a target profit, and the profit which satisfies some chance constraints with at least some given confidence level. A model to help the decision maker in a newsboy problem to assess the value of information is presented by Lee [13] for the potential benefits of demand forecasting to decrease the risk of overstocking or shortage.

Hayya et al. [14] considered the reorder point, order quantity inventory model where the demand and the lead time are independently and identically distributed random variables. They used a normal approximation to show how to obtain regression equations for the optimal cost and the optimal policy parameters in terms of the problem parameters. Dash et al. [15] have discussed the deterministic equivalent of fuzzy chance constraint programming problem in different scenarios for the fuzzy random variable.

$\mathrm{Hu}$ et al. [16] presented fuzzy random models for the newsboy problem in the decentralized and centralized systems facing imperfect quality and the optimal policies for the systems analyzed and derived.

Zhang [17] presented a Lagrangian relaxation approach to solve the multiproduct newsboy problem as a mixed integer nonlinear programming model with both supplier quantity discounts and a budget constraint. The profit for newsboytype product is introducing a new capacity index, which is based on statistical hypothesis testing analyzed by $\mathrm{Su}$ et al. [18].

Kotb and Fergany [19] derived the analytical solution of the EOQ model of multiple items with both demanddependent unit cost and leading time using geometric programming approach by considering continuous functions of demand rate and leading time for the varying purchase and leading time for crashing costs, respectively. They deduced the optimal order quantity, the demand rate, and the leading time as decision variables and also the optimal total cost. Dey and Chakraborty [20] presented a fuzzy random continuous review system with the annual customer demand distributed uniformly and proposed a methodology to minimize the cost.

R. Banerjee and S. Banerjee [21] considered a probabilistic inventory model under probabilistic and imprecise constraints with uniform lead-time demand and fuzzy cost components. Nagare and Dutta [22] captured both fuzzy perception and randomness of customer demand to determine an unambiguous optimal order quantity from a set of $n$ fuzzy observations in a news-vendor inventory setting in the presence of fuzzy random variable demand.

Jahantigh et al. [23] computed the compromised solution of a LR fuzzy linear system by the use of ranking fuzzy numbers. Karpagam and Sumathi [24] used the ranking function to find the fuzzy optimal solution of fully fuzzy linear programming problems.

Dutta and Kumar [25] determined the optimal total cost and the optimal order quantity for an inventory model without shortages in a fuzzy environment. They used signed distance method to compute economic order quantity. This model deals with indeterminacy information for multiproduct newsboy problem, which was initially transformed into deterministic model, and the solution was obtained by classic integer programming method proposed by Ding [26].

Zolfagharinia and Isotupa [27] pointed out some flaws in the simulation model and some of the formulae for inventory level and ordering quantity. Majumder et al. [28] formulated an EPQ model for deteriorating items under partial trade credit policy with crisp and fuzzy demand. They have used weighted sum method to convert a multiobjective to a single objective and the model was solved by Generalized Reduced Gradient method.

A real-world multiperiod inventory control problem under budget constraint is investigated and the weighted linear sum of objectives is applied to generate a singleobjective model for the biobjective problem and a harmony search algorithm is developed to solve the complex inventory problem by Mousavi et al. [29]. The goal is to find the optimal ordered quantities of products; not only the total inventory cost but also the required storage space as a fuzzy number to store the products is minimized.

Soni and Joshi [30] considered a periodic review inventory model in fuzzy-stochastic environment with lead time and the back order rate as control variables and by applying the criterion of credibility they determined the expected shortages.

To capture the uncertainty of demand, Kumar and Goswami [31] employed the fuzzy expectation, signed distance, and possibility or necessity measure to the fuzzy model to transform it into an equivalent deterministic nonlinear programming problem where the model is restricted to budget and allowable shortages.

Kazemi et al. [32] developed and extended a fuzzy inventory model of an existing economic order quantity with back orders in which both demand and lead times are fuzzified to decrease the total inventory cost. Dash and Sahoo [33] obtained the optimum order quantity and the expected profit by using Buckley's concept of minimization of fuzzy numbers for a single-period inventory model.

Nagare et al. [34] developed an inventory model to determine the optimal order quantity and weight factor on the basis of revised forecasts and the results demonstrate economic benefits of using revised demand, negative impact of constraints, and role of demand distribution entropy in determining the order size and expected profit. 
A fuzzy inventory model for fixed deteriorating items with shortages under fully backlogged condition is formulated and solved by Raula et al. [35]. They defuzzified the model by graded unit preference integration method and all related inventory parameters were assumed to be hexagonal fuzzy numbers.

Sangal et al. [36] developed an inventory model for noninstantaneous decaying items under crisp and fuzzy environment. They have considered stock dependent demand rate and variable deterioration and defuzzified the total cost function by signed distance method.

The important feature of the present work is to determine the optimum order quantity by considering a SPIFPM with demand as a fuzzy random variable in the objective function subject to a chance constraint, where the storage space distributed normally and exponentially, which is different from the previous studies. Initially we used the weighted sum method, ranking function to the objective function, and some theorems have been developed to transform the SPIFPM into a crisp model.

The remaining part of the paper is organized as follows. Some basic concepts and results of uncertain theory are given in Section 2. In Section 3, an analytical model is formulated for the SPIFPM under a chance constraint. In Section 4, we present a transformation technique to convert the model to an equivalent deterministic model. In Section 5, a numerical example is given for verification. Finally, in Section 6, some conclusions are listed.

\section{Preliminaries}

From literature study, here we present some basic concepts regarding the uncertainty theory.

Definition 1 (fuzzy set [1]). A fuzzy set $\widetilde{A}$ over a universal set $X$ is defined as a set of ordered pairs $\widetilde{A}=\left\{\left(x, \mu_{A}(x)\right): x \in X\right\}$ and is characterized by its membership function $\mu_{A}(x)$ where $\mu_{A}(x): X \rightarrow[0,1]$ for each $x \in X$.

Definition 2 (triangular fuzzy number [15]). The triangular fuzzy number is a fuzzy number denoted as $\widetilde{A}=\left(A_{1}, A_{2}, A_{3}\right)$ and is interpreted by its membership function as follows:

$$
\mu_{\widetilde{A}}(x)= \begin{cases}\frac{x-A_{1}}{A_{2}-A_{1}}, & \text { if } A_{1} \leq x \leq A_{2} \\ \frac{A_{3}-x}{A_{3}-A_{2}}, & \text { if } A_{2} \leq x \leq A_{3} \\ 0, & \text { otherwise. }\end{cases}
$$

Definition 3 ( $\alpha$-cut of a fuzzy number [15]). The $\alpha$-cut of the fuzzy number $\widetilde{A}$ is the set $\left\{x \mid \mu_{A}(x) \geq \alpha\right\}$ for $\alpha \in(0,1)$ and denoted as $\widetilde{A}[\alpha]$.
Definition 4 (partial order relation of two fuzzy numbers [9]). Let $\widetilde{A}=\left(A_{1}, A_{2}, A_{3}\right)$ and $\widetilde{B}=\left(B_{1}, B_{2}, B_{3}\right)$ be two fuzzy numbers with $\alpha$-cut $\widetilde{A}[\alpha]=\left[A_{*}, A^{*}\right]$ and $\widetilde{B}[\alpha]=\left[B_{*}, B^{*}\right]$, respectively; then $\widetilde{A} \preceq \widetilde{B}$ if and only if $A^{*} \leq B_{*}$ and $\widetilde{A} \succeq \widetilde{B}$ if and only if $A_{*} \leq B^{*}$.

Definition 5 (fuzzy random variable $[3,6]$ ). The random variables whose parameters like mean, variance, and so forth are fuzzy numbers are called fuzzy random variables. A fuzzy random variable $\widetilde{X}$ with mean $\widetilde{m}_{x}$ and variance $\widetilde{\sigma}_{x}^{2}$ is denoted by $\widetilde{X}\left(\widetilde{m}, \widetilde{\sigma}^{2}\right)$.

Definition 6 (mean of a continuous fuzzy random variable [6]). Let $\widetilde{U}=a_{1} \widetilde{X}_{1} \oplus a_{2} \widetilde{X}_{2} \oplus \cdots \oplus a_{n} \widetilde{X}_{n}$ be a FRV, which is the linear combination of $n$ number of independent fuzzy random variables $\widetilde{X}_{1}, \widetilde{X}_{2}, \ldots, \widetilde{X}_{n}$, whose means are fuzzy numbers $\widetilde{m}_{X_{1}}, \widetilde{m}_{X_{2}}, \ldots, \widetilde{m}_{X_{n}}$, respectively. Then $\widetilde{m}_{U}[\alpha]=$ $\sum_{j=1}^{n} a_{j} \widetilde{m}_{X_{j}}[\alpha]$.

Definition 7 (variance of a continuous fuzzy random variable [6]). Let $\widetilde{U}=a_{1} \widetilde{X}_{1} \oplus a_{2} \widetilde{X}_{2} \oplus \cdots \oplus a_{n} \widetilde{X}_{n}$ be the linear combination of $n$ number of independent fuzzy random variables $\widetilde{X}_{1}, \widetilde{X}_{2}, \ldots, \widetilde{X}_{n}$, whose variances are the fuzzy numbers $\widetilde{\sigma}_{X_{1}}^{2}, \widetilde{\sigma}_{X_{2}}^{2}, \ldots, \widetilde{\sigma}_{X_{n}}^{2}$, respectively. Then $\widetilde{\sigma}_{U}^{2}[\alpha]=\sum_{j=1}^{n} a_{j}^{2} \widetilde{\sigma}_{X_{j}}^{2}[\alpha]$.

Definition 8 (ranking function $[23,24]$ ). The ranking function is defined on a set of real numbers $(R)$, which maps each fuzzy number into the real line, where a natural order exists; that is, $R: F(R) \rightarrow R$, where $F(R)$ is a set of fuzzy numbers. To calculate the triangular weights, ranking function is effective. Let $\widetilde{a}=(a, b, c)$ be a triangular fuzzy number. We define a standard ranking function as $R(\widetilde{a})=(a+2 * b+c) / 4$.

Definition 9 (weighted sum method [28]). This method can be interpreted by some positive weights or priority assigned to the objective criterion by the decision maker. Here we express the weighted sum method by multiplying the weights by the users, each supplied in the objective, and the weights are chosen in a way such that their sum is one; that is,

$$
\text { optimize } \sum_{i=1}^{n} w_{i} f_{i}(x), \quad w_{i} \in[0,1], x \in X
$$

such that $\sum_{i=1}^{n} w_{i}=1$, where $w_{i}$ are the weights of the $i$ th objective functions.

Definition 10 (fuzzy normal distribution [15]). A normal fuzzy random variable $N\left(\widetilde{m}, \widetilde{\sigma}^{2}\right)$ is a normally distributed random variable with fuzzy mean $\widetilde{m}$ and fuzzy variance $\widetilde{\sigma}^{2}$ as fuzzy parameters. Let $N\left(m, \sigma^{2}\right)$ denote the crisp normal random variable with mean $m$ and variance $\sigma^{2}$ and let $f\left(x, m, \sigma^{2}\right)$ be the density function of the crisp normal distribution where

$$
f\left(x, m, \sigma^{2}\right)=\frac{1}{\sqrt{2 \pi} \sigma} e^{-(x-m)^{2} / 2 \sigma^{2}}, \quad \sigma>0,
$$


so $(1 / \sqrt{2 \pi} \sigma) \int_{-\infty}^{\infty} e^{-(x-m)^{2} / 2 \sigma^{2}} d x=1$. Now the fuzzy probability of the FRV $\widetilde{X}$ on the interval $[c, d]$ is a fuzzy number whose $\alpha$-cut is

$$
\begin{aligned}
& \widetilde{P}(c \leq \widetilde{X} \leq d)[\alpha]=\left\{\frac{1}{\sqrt{2 \pi} \sigma} \int_{c}^{d} e^{-(x-m)^{2} / 2 \sigma^{2}} d x \mid m\right. \\
& \left.\in \widetilde{m}[\alpha], \sigma^{2} \in \widetilde{\sigma}^{2}[\alpha]\right\}=\left\{\frac{1}{\sqrt{2 \pi}} \int_{z_{1}}^{z_{2}} e^{-z^{2} / 2} d z \mid m\right. \\
& \left.\in \widetilde{m}[\alpha], \sigma^{2} \in \widetilde{\sigma}^{2}[\alpha]\right\},
\end{aligned}
$$

where $z_{1}=(c-m) / \sigma$ and $z_{2}=(d-m) / \sigma$.

Definition 11 (fuzzy exponential distribution [15]). Let $\widetilde{X}$ be a exponentially distributed fuzzy random variable with density function $f(x, \widetilde{\lambda})$ being a fuzzy set and its $\alpha$-cut is

$$
f(x ; \tilde{\lambda})[\alpha]=\left\{\tilde{\lambda} e^{-\tilde{\lambda} x} \mid \lambda \in \tilde{\lambda}(\alpha)\right\}
$$

Now the fuzzy probability of $\widetilde{X}$ on the interval $[c, d]$ is a fuzzy number and denoted by $\widetilde{P}(c \leq \widetilde{X} \leq d)$, where $P_{*}(\alpha)=$ $\min \left\{\int_{c}^{d} \lambda e^{-\lambda x} d x \mid \lambda \in \tilde{\lambda}[\alpha]\right\}$ and $P^{*}(\alpha)=\max \left\{\int_{c}^{d} \lambda e^{-\lambda x} d x \mid\right.$ $\lambda \in \tilde{\lambda}[\alpha]\}$.

Definition 12 (Fuzzy Chance Constrained Programming problem (FCCP) [26]). A general chance constraint programming problem CCP is of the following form:

$$
\begin{array}{ll}
\text { (CCP): Optimize } & \sum_{j=1}^{k} c_{j} x_{j} \\
\text { Subject to } & P\left(\sum_{j=1}^{k} a_{i j} x_{j} \leq b_{i}\right) \geq \gamma_{i} \\
& \sum_{j=1}^{k} b_{l j} x_{j} \geq h_{l} \\
& x_{j} \geq 0, \\
& b_{l j}, h_{l} \in R, \\
& i=1,2, \ldots, m, \\
& l=1,2, \ldots, n, \\
& 0 \leq \gamma_{i} \leq 1,
\end{array}
$$

where at least one of $a_{i j}, c_{j}$, and $b_{i}$ is treated as random variable.
So FCCP is a CCP given as above form where at least one of $a_{i j}, c_{j}$, and $b_{i}$ is treated as FRV.

\section{Mathematical Formulation}

Notations used in the proposed model are presented in Notations section.

3.1. Assumptions. We imposed the following assumptions to develop the model.

(i) For all $j, \widetilde{D}_{j}$ are independent fuzzy random variables whose mean $\widetilde{\mu}_{D}$ are triangular fuzzy numbers.

(ii) For all $j, \widetilde{W}_{j}$ are independent fuzzy random variables whose mean $\tilde{\mu}_{W}$ and variance $\widetilde{\sigma}_{W}^{2}$ are triangular fuzzy numbers.

(iii) The time period is precise for all $j$, that is, single period.

(iv) The decision maker's order quantity $Q_{j}$ is optimal for all products at the beginning of the period.

(v) At the end of the season the leftover items are salvaged.

(vi) The lost sales penalty cost is zero.

3.2. Single-Period Inventory Fuzzy Probabilistic Model (SPIFPM). The profit function $Z_{j}$ associated with each order quantity $Q_{j}$ and fuzzy demand $\widetilde{D}_{j}$ is given as follows:

$$
\begin{aligned}
Z_{j}\left(Q_{j}, \widetilde{D}_{j}\right)= & p_{j} \min \left\{Q_{j}, \widetilde{D}_{j}\right\}+s_{j} \max \left\{Q_{j}-\widetilde{D}_{j}, 0\right\} \\
& -c_{j} Q_{j} ; \quad j=1,2, \ldots, k .
\end{aligned}
$$

Here two situations are explored to discuss the profit function, that is, overstock situation $\left(\widetilde{D}_{j} \leq Q_{j}\right)$ and understock situation $\left(\widetilde{D}_{j} \geq Q_{j}\right)$. Then the above equation boils down to the following form:

$$
\begin{aligned}
& Z_{j}\left(Q_{j}, \widetilde{D}_{j}\right) \\
& = \begin{cases}\widetilde{Z}_{j}\left(Q_{j}, \widetilde{D}_{j}\right)=\left(p_{j}-s_{j}\right) \widetilde{D}_{j}+\left(s_{j}-c_{j}\right) Q_{j}, & \widetilde{D}_{j} \leq Q_{j} \\
\widetilde{Z}_{j}\left(Q_{j}, \widetilde{D}_{j}\right)=\left(p_{j}-c_{j}\right) Q_{j}, & \widetilde{D}_{j} \geq Q_{j},\end{cases}
\end{aligned}
$$

where $\bar{Z}_{j}$ and $\underline{Z}_{j}$ are profit functions for each product in overstock and understock situations, respectively. Due to fuzziness of demand $\widetilde{D}_{j}$, we get either a fuzzy overstock profit $\widetilde{\bar{Z}}_{j}\left(Q_{j}, \widetilde{D}_{j}\right)$ or a fuzzy understock profit $\underline{Z}_{j}\left(Q_{j}, \widetilde{D}_{j}\right)$. Hence the total expected profit $\widetilde{Z}_{j}\left(Q_{j}, \widetilde{D}_{j}\right)$ corresponding to both overstock and understock situations is given as follows:

$$
\widetilde{Z}_{j}\left(Q_{j}, \widetilde{D}_{j}\right)=\widetilde{\bar{Z}}_{j}\left(Q_{j}, \widetilde{D}_{j}\right) \cup \underline{\widetilde{Z}}_{j}\left(Q_{j}, \widetilde{D}_{j}\right) .
$$


Thus a multi-item SPIFPM subject to a storage space chance constraint with a confidence level $\gamma$ can be stated as follows:

$$
\begin{array}{ll}
\text { (SPIFPM): Maximize } & \widetilde{Z}\left(Q_{1}, Q_{2}, \ldots, Q_{k}\right) \\
& =\sum_{j=1}^{k} \widetilde{Z}_{j}\left(Q_{j}, \widetilde{D}_{j}\right) \\
\text { subject to } \quad & \widetilde{P}\left(\sum_{j=1}^{\widetilde{k}} a_{j} Q_{j} \leq \widetilde{W}\right) \geq \widetilde{\gamma} \\
& Q_{j} \geq 0 \\
& \text { for } j=1,2, \ldots, k .
\end{array}
$$

In the above model, we consider demand $\widetilde{D}_{j}$ as a FRV present in the objective function and the total storage space $\widetilde{W}$ are independent FRVs present in the constraint distributed normally and exponentially.

\section{Transformation Technique}

4.1. Crisp Equivalent of the Fuzzy Objective Function. In SPIM, the defined resultant profit function is fuzzy, since the customer demand for each product is imprecise. Now our objective is to convert the fuzzy model to crisp model. Using the weighted function to the profit function, we have

$$
\begin{aligned}
& \widetilde{Z}_{j}\left(Q_{j}, \widetilde{D}_{j}\right)= \omega \widetilde{\bar{Z}}_{j}\left(Q_{j}, \widetilde{D}_{j}\right)+(1-\omega) \widetilde{Z}_{j}\left(Q_{j}, \widetilde{D}_{j}\right) \Longrightarrow \\
& \widetilde{Z}_{j}\left(Q_{j}, \widetilde{D}_{j}\right)=\omega\left[\sum_{j=1}^{k}\left(p_{j}-s_{j}\right) \widetilde{D}_{j}+\left(s_{j}-c_{j}\right) Q_{j}\right] \\
&+(1-\omega)\left[\sum_{j=1}^{k}\left(p_{j}-c_{j}\right) Q_{j}\right] .
\end{aligned}
$$

Here $\widetilde{D}_{j}$ are FRVs whose mean $\widetilde{m_{D_{j}}}$ are triangular fuzzy numbers. To make it crisp apply the ranking function as $R\left(\overline{m_{D_{j}}}\right)=\left(m_{D_{j}}+2 m_{D_{j}}+\overline{m_{D_{j}}}\right) / 4$. So the above profit function becomes

$$
\begin{aligned}
& \widetilde{Z}_{j}\left(Q_{j}, \widetilde{D}_{j}\right)=\omega\left[\sum_{j=1}^{k} \frac{\left(p_{j}-s_{j}\right)\left(\frac{\left.m_{D_{j}}+2 m_{D_{j}}+\overline{m_{D_{j}}}\right)}{4}\right.}{\left.+\left(s_{j}-c_{j}\right) Q_{j}\right]+(1-\omega)\left[\sum_{j=1}^{k}\left(p_{j}-c_{j}\right) Q_{j}\right] .}\right.
\end{aligned}
$$

Therefore the crisp equivalent of the objective function is as follows:

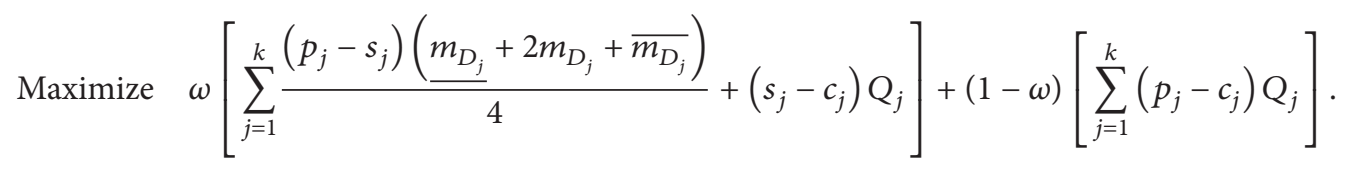

4.2. Crisp Equivalent of the Fuzzy Chance Constraint. The following results are proved to convert the fuzzy chance constraint to a deterministic form.

Theorem 13 (Case 1 ( $\widetilde{W}$ is normally distributed fuzzy random variable)). If $\widetilde{W}$ is a normally distributed FRV, $a_{j} \in R$ and, with predetermined confidence level $\gamma$, the inequality $\widetilde{P}\left(\sum_{j=1}^{k} a_{j} Q_{j} \leq \widetilde{W}\right) \geq \widetilde{\gamma}$ is equivalent to

$$
\sum_{j=1}^{k} a_{j} Q_{j} \leq \mu_{W *}(\alpha)+\sigma_{W *}(\alpha) F^{-1}\left(1-\gamma^{*}(\alpha)\right)
$$

for $\alpha \in[0,1]$ and $F$ is the cumulative distribution function of $N(0,1)$ distribution.

Proof. Let us consider the constraint of the model as follows:

$$
\widetilde{P}\left(\sum_{j=1}^{k} a_{j} Q_{j} \leq \widetilde{W}\right) \geq \widetilde{\gamma}, \quad a_{j} \in R
$$

where $\widetilde{W}$ is a normally distributed FRV whose mean and variance are fuzzy numbers denoted by $\tilde{\mu}_{W}$ and $\widetilde{\sigma}_{W}^{2}$ and their $\alpha$-cuts are $\tilde{\mu}_{W}[\alpha]=\left[\mu_{W *}(\alpha), \mu_{W}^{*}(\alpha)\right]$ and $\tilde{\sigma}_{W}^{2}[\alpha]=$ $\left[\sigma_{W *}^{2}(\alpha), \sigma_{W}^{2}{ }^{*}(\alpha)\right]$, respectively. The $\alpha$-cut of $\tilde{\gamma}$ is $\tilde{\gamma}[\alpha]=$ $\left[\gamma_{*}(\alpha), \gamma^{*}(\alpha)\right]$

Let $\sum_{j=1}^{k} a_{j} Q_{j}=U$. Here $U$ is a real number, since $a_{j}$, $j=1,2, \ldots, k$, are real numbers. Then the above inequality can be written as $P(U \leq \widetilde{W}) \geq \widetilde{\gamma}$. Here $\widetilde{W}$ is a FRV whose mean and variance are the fuzzy numbers $\tilde{\mu}_{W}$ and $\tilde{\sigma}_{W}^{2}$, which can be computed from the crisp random variable $W$, that is, $W \in \widetilde{W}[\alpha]$. That means $\mu_{W} \in \widetilde{\mu}_{W}[\alpha]$ and $\sigma_{W}^{2} \in \widetilde{\sigma}_{W}^{2}[\alpha]$ which satisfy $\left(1 / \sqrt{2 \pi} \sigma_{W}\right) \int_{-\infty}^{\infty} e^{-\left(W-\mu_{W}\right)^{2} / 2 \sigma_{W}^{2}} d W=1$.

Now the $\alpha$-cut of the probability $\widetilde{P}\left(\sum_{j=1}^{k} a_{j} Q_{j} \leq \widetilde{W}\right)$ is

$$
\begin{aligned}
& \widetilde{P}\left(\sum_{j=1}^{k} a_{j} Q_{j} \leq \widetilde{W}\right)[\alpha]=\left\{P(U \leq W) \mid \mu_{W}\right. \\
& \left.\quad \in \widetilde{\mu}_{W}[\alpha], \sigma_{W}^{2} \in \widetilde{\sigma}_{W}^{2}[\alpha]\right\}=\left\{1-P(W \leq U) \mid \mu_{W}\right.
\end{aligned}
$$




$$
\begin{aligned}
& \left.\in \tilde{\mu}_{W}[\alpha], \sigma_{W}^{2} \in \widetilde{\sigma}_{W}^{2}[\alpha]\right\}=\{1 \\
& -\frac{1}{\sqrt{2 \pi} \sigma_{W}} \int_{-\infty}^{U} e^{-\left(W-\mu_{W}\right)^{2} / 2 \sigma_{W}^{2}} d W \mid \mu_{W} \\
& \left.\in \widetilde{\mu}_{W}[\alpha], \sigma_{W}^{2} \in \widetilde{\sigma}_{W}^{2}[\alpha]\right\} .
\end{aligned}
$$

Let $V=\left(W-\mu_{W}\right) / \sigma_{W}$. Then

$$
\begin{aligned}
& \widetilde{P}\left(\sum_{j=1}^{k} a_{j} Q_{j} \leq \widetilde{W}\right)[\alpha]=\{1 \\
& \quad-\frac{1}{\sqrt{2 \pi}} \int_{-\infty}^{\left(U-\mu_{W}\right) / \sigma_{W}} e^{-V^{2} / 2} d V \mid \mu_{W} \in \widetilde{\mu}_{W}[\alpha], \sigma_{w}^{2} \\
& \left.\quad \in \widetilde{\sigma}_{w}^{2}[\alpha]\right\}=\left\{1-F\left(\frac{U-\mu_{W}}{\sigma_{W}}\right) \mid \mu_{W}\right. \\
& \left.\quad \in \widetilde{\mu}_{W}[\alpha], \sigma_{W}^{2} \in \widetilde{\sigma}_{W}^{2}[\alpha]\right\},
\end{aligned}
$$

where $F$ is the cumulative distribution function of $N(0,1)$ distribution which is an increasing function. So

$$
\begin{aligned}
& \min \left\{1-F\left(\frac{U-\mu_{W}}{\sigma_{W}}\right) \mid \mu_{W} \in \widetilde{\mu}_{W}[\alpha], \sigma_{W}^{2}\right. \\
& \left.\in \widetilde{\sigma}_{W}^{2}[\alpha]\right\}=1-F\left(\frac{U-\mu_{W *}(\alpha)}{\sigma_{W *}(\alpha)}\right),
\end{aligned}
$$

$$
\begin{aligned}
& \max \left\{1-F\left(\frac{U-\mu_{W}}{\sigma_{W}}\right) \mid \mu_{W} \in \tilde{\mu}_{W}[\alpha], \sigma_{W}^{2}\right. \\
& \left.\in \widetilde{\sigma}_{W}^{2}[\alpha]\right\}=1-F\left(\frac{U-\mu_{W}^{*}(\alpha)}{\sigma_{W}^{*}(\alpha)}\right) .
\end{aligned}
$$

So

$$
\begin{aligned}
& \widetilde{P}\left(\sum_{j=1}^{k} a_{j} Q_{j} \leq \widetilde{W}\right)[\alpha] \\
& \quad=\left[1-F\left(\frac{U-\mu_{W *}(\alpha)}{\sigma_{W *}(\alpha)}\right), 1-F\left(\frac{U-\mu_{W}^{*}(\alpha)}{\sigma_{W}^{*}(\alpha)}\right)\right] .
\end{aligned}
$$

Using the Inequality law due to Nanda et al. [9], the constraint $\widetilde{P}\left(\sum_{j=1}^{k} a_{j} Q_{j} \leq \widetilde{W}\right) \geq \widetilde{\gamma}$ becomes

$$
\begin{aligned}
& 1-F\left(\frac{U-\mu_{W *}(\alpha)}{\sigma_{W *}(\alpha)}\right) \geq \gamma^{*}(\alpha) \Longrightarrow \\
& \sum_{j=1}^{k} a_{j} Q_{j} \leq \mu_{W *}(\alpha)+\sigma_{W *}(\alpha) F^{-1}\left(1-\gamma^{*}(\alpha)\right) .
\end{aligned}
$$

Hence the proof is complete.

Therefore, finally the SPIFPM is transformed to an equivalent single-period inventory crisp model (SPICM) as follows:

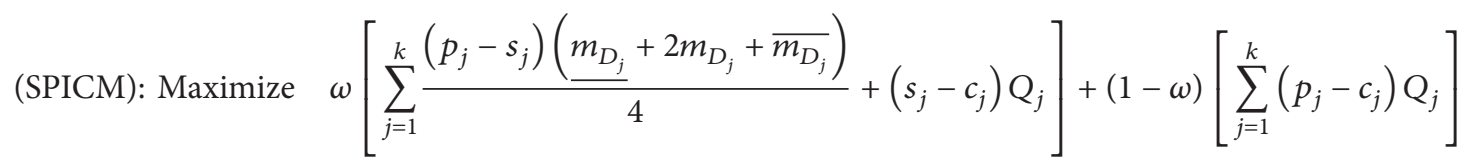

$$
\begin{aligned}
& \text { Subject to } \sum_{j=1}^{k} a_{j} Q_{j} \leq \mu_{W *}(\alpha)+\sigma_{W *}(\alpha) F^{-1}\left(1-\gamma^{*}(\alpha)\right) \\
& Q_{j} \geq 0 \\
& \text { for } \alpha \in[0,1] \text {, } \\
& j=1,2, \ldots, k \text {. }
\end{aligned}
$$

Theorem 14 (Case 2 ( $\widetilde{W}$ follows fuzzy exponential distribution)). If $\widetilde{W}$ is an exponentially distributed FRV with fuzzy parameter $\widetilde{\lambda}, a_{j} \in R$, then $\widetilde{P}\left(\sum_{j=1}^{k} a_{j} Q_{j} \leq \widetilde{W}\right) \geq \widetilde{\gamma}$ is equivalent to $e^{-\lambda^{*}(\alpha) U} \geq \gamma^{*}(\alpha)$ for each $\alpha \in[0,1]$, where $\tilde{\lambda}[\alpha]=$ $\left[\lambda_{*}(\alpha), \lambda^{*}(\alpha)\right]$.

Proof. The $\alpha$-cut of the fuzzy probability $\widetilde{P}\left(\sum_{j=1}^{k} a_{j} Q_{j} \leq \widetilde{W}\right)$ is

$$
\begin{aligned}
& \widetilde{P}\left(\sum_{j=1}^{k} a_{j} Q_{j} \leq \widetilde{W}\right)[\alpha]=\{P(U \leq W) \mid W \in \widetilde{W}[\alpha]\} \\
& \quad=\left\{\int_{U}^{\infty} \lambda e^{-\lambda W} d W \mid \lambda \in \widetilde{\lambda}[\alpha]\right\}
\end{aligned}
$$

respectively.

where $P_{*}(\alpha)$ and $P^{*}(\alpha)$ are the solutions of the optimization problems

$$
\begin{aligned}
& \underset{\lambda_{*}(\alpha) \leq \lambda \leq \lambda^{*}(\alpha)}{\operatorname{minimize}} e^{-\lambda U}, \\
& \underset{\lambda_{*}(\alpha) \leq \lambda \leq \lambda^{*}(\alpha)}{\operatorname{maximime}} e^{-\lambda U},
\end{aligned}
$$


Here $e^{-\lambda U}$ attains its minimum at $\lambda^{*}(\alpha)$ and maximum at $\lambda_{*}(\alpha)$, since $e^{-\lambda U}$ is a decreasing function of $\lambda$. That is, $\min e^{-\lambda U}=\lambda^{*}(\alpha)$ and $\max e^{-\lambda U}=\lambda^{*}(\alpha)$.

Hence

$$
\widetilde{P}\left(\sum_{j=1}^{k} a_{j} Q_{j} \leq \widetilde{W}\right)[\alpha]=\left[e^{-\lambda^{*}(\alpha) U}, e^{-\lambda_{*}(\alpha) U}\right] .
$$

Therefore the deterministic equivalent of the fuzzy chance constraint

$$
\begin{aligned}
& \widetilde{P}\left(\sum_{j=1}^{k} a_{j} Q_{j} \leq \widetilde{W}\right) \geq \tilde{\gamma} \\
& \text { becomes } e^{-\lambda^{*}(\alpha) U} \geq \gamma^{*}(\alpha) .
\end{aligned}
$$

\section{Numerical Example}

Let us consider a SPIFPM to illustrate the effectiveness of the above approach with the following data:

$$
\begin{gathered}
\text { (SPIFPM): Maximize }\left[\sum_{j=1}^{2}\left(p_{j}-s_{j}\right) \widetilde{D}_{j}+\left(s_{j}-c_{j}\right) Q_{j}\right] \\
\cup\left[\sum_{j=1}^{2}\left(p_{j}-c_{j}\right) Q_{j}\right]
\end{gathered}
$$

$$
\begin{array}{ll}
\text { Subject to } & \widetilde{P}\left(4 Q_{1} \oplus 3 Q_{2} \leq \widetilde{W}\right) \geq \widetilde{0.5} \\
& Q_{1} \geq 0, \\
& Q_{2} \geq 0,
\end{array}
$$

where the other data for multi-items is given in Table 1 .

Here $\widetilde{D}_{1}, \widetilde{D}_{2}$ are independent normally distributed FRVs with mean $\widetilde{\mu}_{D_{1}}, \widetilde{\mu}_{D_{2}}$ being fuzzy numbers. Also $\widetilde{W}$ are independent normally distributed FRVs with mean $\widetilde{\mu}_{W}$ and variance $\widetilde{\sigma}_{W}^{2}$ as fuzzy numbers and $\widetilde{0.5}$ is also a fuzzy number. All these fuzzy numbers are assumed to be linear triangular as follows: $\widetilde{\mu}_{D_{1}}=\langle 200 / 300 / 400\rangle, \widetilde{\mu}_{D_{2}}=\langle 700 / 800 / 900\rangle$, $\widetilde{\mu}_{W}=\langle 2000 / 2010 / 2020\rangle, \widetilde{\sigma}_{W}^{2}=\langle 300 / 400 / 500\rangle$, and $\widetilde{0.5}=$ $\langle 0.4 / 0.5 / 0.6\rangle$.

5.1. Solution. Initially applying the weighted sum method to the objective function, we can write

$$
\begin{aligned}
\text { Maximize } & \widetilde{Z}\left(Q_{1}, Q_{2}\right) \\
= & \omega\left[\sum_{j=1}^{2}\left(p_{j}-s_{j}\right) \widetilde{D}_{j}+\left(s_{j}-c_{j}\right) Q_{j}\right] \\
& +(1-\omega)\left[\sum_{j=1}^{2}\left(p_{j}-c_{j}\right) Q_{j}\right] .
\end{aligned}
$$

TABLE 1

\begin{tabular}{lcccc}
\hline Product name & $c_{j}$ & $p_{j}$ & $s_{j}$ & $a_{j}$ \\
\hline Product 1 & 30 & 34 & 28 & 4 \\
Product 2 & 40 & 43 & 35 & 3 \\
\hline
\end{tabular}

Let $\omega=0.6$ and using the above cost data to the above expression we have

$$
\begin{array}{ll}
\text { Maximize } & \widetilde{Z}\left(Q_{1}, Q_{2}\right) \\
& =0.4 Q_{1}-1.8 Q_{2}+3.6 \widetilde{D}_{1}+4.8 \widetilde{D}_{2} .
\end{array}
$$

Here $\widetilde{D}_{1}, \widetilde{D}_{2}$ are FRVs whose mean $\widetilde{\mu}_{D_{1}}, \widetilde{\mu}_{D_{2}}$ are triangular fuzzy numbers with the $\alpha$-cuts being

$$
\begin{aligned}
& \tilde{\mu}_{D_{1}}[\alpha]=[200+100 \alpha, 400-100 \alpha], \\
& \tilde{\mu}_{D_{2}}[\alpha]=[700+100 \alpha, 900-100 \alpha] .
\end{aligned}
$$

Applying the ranking function to the above objective function we have

$$
\text { Maximize } \begin{aligned}
\widetilde{Z} & \left(Q_{1}, Q_{2}\right) \\
= & 0.4 Q_{1}-1.8 Q_{2}+3.6 R\left(\widetilde{m}_{D_{1}}\right) \\
& +4.8 R\left(\widetilde{m}_{D_{2}}\right) .
\end{aligned}
$$

Hence the crisp equivalent of the objective function takes the following form:

$$
\text { Maximize } \quad 0.4 Q_{1}-1.8 Q_{2}+4920 .
$$

Now let us consider the constraint $\widetilde{P}\left(4 Q_{1}+3 Q_{2} \leq \widetilde{W}\right) \geq \widetilde{0.5}$. We can convert this constraint to its deterministic form as $\widetilde{W}$ follows normal and exponential distribution.

Case 1 ( $\widetilde{W}$ is normally distributed fuzzy random variable). As the mean $\widetilde{\mu}_{W}$, variance $\widetilde{\sigma}_{W}^{2}$ and $\widetilde{0.5}$ are triangular fuzzy numbers; their $\alpha$-cuts are

$$
\begin{aligned}
\widetilde{\mu}_{W}[\alpha] & =[2000+10 \alpha, 2020-10 \alpha], \\
\widetilde{\sigma}_{W}^{2} & =[300+100 \alpha, 500-100 \alpha], \\
\widetilde{0.5}[\alpha] & =[0.4+\alpha, 0.6-\alpha] .
\end{aligned}
$$

Let $4 Q_{1}+3 Q_{2}=U$. So the constraint is given by $\widetilde{P}(U \leq \widetilde{W}) \geq$ $\widetilde{0.5}$. Using the above theorem result, the $\alpha$-cut of $\widetilde{P}(U \leq \widetilde{W})$ becomes

$$
\begin{aligned}
\widetilde{P} & (U \leq \widetilde{W})[\alpha] \\
& =\left[1-F\left(\frac{U-\mu_{W *}(\alpha)}{\sigma_{W *}(\alpha)}\right), 1-F\left(\frac{U-\mu_{W}^{*}(\alpha)}{\sigma_{W}^{*}(\alpha)}\right)\right] .
\end{aligned}
$$

So the crisp conversion of the constraint is

$$
1-F\left(\frac{U-\mu_{W *}(\alpha)}{\sigma_{W *}(\alpha)}\right) \geq 0.6-0.1 \alpha
$$


which can be further simplified as

$$
\begin{aligned}
& U-\mu_{W *}(\alpha) \leq \sigma_{W *}(\alpha) F^{-1}(0.6+0.1 \alpha) \Longrightarrow \\
& 4 Q_{1}+3 Q_{2} \leq 5+\alpha+(\sqrt{3+\alpha}) F^{-1}(0.4+0.1 \alpha) .
\end{aligned}
$$

Hence the crisp equivalent, that is, SPICM of the original SPIFPM, takes the following form:

$$
\begin{array}{ll}
\text { (SPICM): Maximize } & 0.4 Q_{1}-1.8 Q_{2}+4920 \\
\text { Subject to } \quad & 4 Q_{1}+3 Q_{2} \\
& \leq 5+\alpha \\
& +(\sqrt{3+\alpha}) F^{-1}(0.4+0.1 \alpha) \\
& Q_{1} \geq 0 \\
& Q_{2} \geq 0 .
\end{array}
$$

Now $F^{-1}(0.4+0.1 \alpha)$ can be found from the normal distribution table. For each $\alpha$ there exists a solution of the above problem. For example, if $\alpha=0.4$, then SPICM reduces to the following nonlinear programming problem:

$$
\begin{array}{cl}
\text { (SPICM): Maximize } & 0.4 Q_{1}-1.8 Q_{2}+4920 \\
\text { Subject to } & \left(4 Q_{1}+3 Q_{2}-2004\right)^{2} \\
& \leq 7.75234 \\
& Q_{1} \geq 0, \\
& Q_{2} \geq 0 .
\end{array}
$$

Using any optimization software its local solution can be determined. Here we have solved this by using LINGO and its solution is $Q_{1}=501.6959$ and $Q_{2}=0$ and the corresponding objective value is 5120.678 . Each value of $\alpha$ gives a corresponding solution.

Case 2 ( $\widetilde{W}$ follows fuzzy exponential distribution). Let $\widetilde{W}$ be an exponential FRV with the parameter $\tilde{\lambda}=\langle 1000,1500$, $2000\rangle$ having $\alpha$-cut as $\tilde{\lambda}[\alpha]=[1000+500 \alpha, 2000-500 \alpha]$. Let $4 Q_{1}+3 Q_{2}=U$. So the constraint is given by $\widetilde{P}(U \leq \widetilde{W}) \geq \widetilde{0.5}$. Using theorem, the crisp conversion of the constraint is

$$
\exp \left(-(2000-500 \alpha)\left(4 Q_{1}+3 Q_{2}\right)\right) \geq 0.6-0.1 \alpha
$$

Hence the crisp equivalent, that is, SPICM of the original SPIFPM, takes the following form:

$$
\begin{aligned}
\text { (SPICM): Maximize } & 0.4 Q_{1}-1.8 Q_{2}+4920 \\
\text { Subject to } & \exp \left(-(2000-500 \alpha)\left(4 Q_{1}+3 Q_{2}\right)\right) \\
& \geq 0.6-0.1 \alpha \\
& Q_{1} \geq 0 \\
& Q_{2} \geq 0 .
\end{aligned}
$$

For each $\alpha$ there exists a solution of the above problem. For example, if $\alpha=0.4$, then SPICM reduces to the following nonlinear programming problem:

$$
\begin{array}{cl}
\text { (SPICM): Maximize } & 0.4 Q_{1}-1.8 Q_{2}+4920 \\
\text { Subject to } & \exp \left(-1800\left(4 Q_{1}+3 Q_{2}\right)\right) \\
& \geq 0.56 \\
& Q_{1} \geq 0 \\
& Q_{2} \geq 0
\end{array}
$$

Using any optimization software its local solution can be determined. Here we have solved this by using LINGO and its solution is $Q_{1}=0.8053035 E-04$ and $Q_{2}=0$ and the corresponding objective value is 4920 . Each value of $\alpha$ gives a corresponding solution.

\section{Conclusion}

This paper presents a solution procedure for solving SPIFPM in which the the storage space in the constraint is a FRV considered under two cases, that is, normal distribution and exponential distribution. Also the demand is a FRV whose mean and variance are fuzzy numbers in the objective function of the given model. Initially some theorems are proposed to transform the fuzzy model into an equivalent crisp model. Then the resultant model is solved by using the LINGO software. When the storage space in the constraint is normally distributed, then the optimal solution is $Q_{1}=$ 501.6959 and $Q_{2}=0$ and the corresponding objective value is 5120.678 and for exponentially distributed random variable its solution is $Q_{1}=0.8053035 E-04$ and $Q_{2}=0$ and the corresponding objective value is 4920 for one value of $\alpha$. However, more approximate solution can be obtained by taking more numbers of values of $\alpha$. For the future scope of the present work, triangular fuzzy number can be replaced by other fuzzy numbers and the demand for each items can be considered as other types of nonindependent FRVs instead of normally distributed independent FRVs. A multiconstrained optimization model can be developed of our present work and our model can be extended to other mathematical models. To analyze the effect of changes in the optimal solution with respect to change in various parameters, sensitivity analysis can be carried out.

\section{Notations}

$Q_{j}$ : Order quantity for item $j$

$\widetilde{D}_{j}$ : Demand as a fuzzy random variable for item $j$

$c_{j}$ : Purchase cost per unit of item $j$

$p_{j}$ : Selling price per unit of item $j$

$s_{j}$ : Salvage value per unit of item $j$

$a_{j}$ : Area required for each unit of item $j$

$\widetilde{Z}_{j}$ : Fuzzy profit function for product $j$

$\widetilde{Z}: \quad$ Fuzzy total profit function for all products

$\widetilde{W}$ : Available storage space, a fuzzy random variable. 


\section{Competing Interests}

The authors declare that they have no competing interests.

\section{References}

[1] L. A. Zadeh, "Fuzzy sets," Information and Computation, vol. 8, no. 3, pp. 338-353, 1965.

[2] J. J. Buckley, Fuzzy Probabilities. New Approach and Applications, Physica, Heidelberg, Germany, 2003.

[3] H. Kwakernaak, "Fuzzy random variables-I. definitions and theorems," Information Sciences, vol. 15, no. 1, pp. 1-29, 1978.

[4] H.-J.Zimmermann, "Fuzzy mathematical programming," Computers \& Operations Research, vol. 10, no. 4, pp. 291-298, 1983.

[5] S. Nanda and K. Kar, "Convex fuzzy mappings," Fuzzy Sets and Systems, vol. 48, no. 1, pp. 129-132, 1992.

[6] M. L. Puri and D. A. Ralescu, "Fuzzy random variables," Journal of Mathematical Analysis and Applications, vol. 114, no. 2, pp. 409-422, 1986

[7] D. Petrović, R. Petrović, and M. Vujošević, "Fuzzy models for the newsboy problem," International Journal of Production Economics, vol. 45, no. 1-3, pp. 435-441, 1996.

[8] K. Das, T. K. Roy, and M. Maiti, "Multi-item stochastic and fuzzy-stochastic inventory models under two restrictions," Computers and Operations Research, vol. 31, no. 11, pp. 17931806, 2004.

[9] S. Nanda, G. Panda, and J. K. Dash, "A new methodology for crisp equivalent of fuzzy chance constrained programming problem," Fuzzy Optimization and Decision Making, vol. 7, no. 1, pp. 59-74, 2008.

[10] N. K. Mahapatra and M. Maiti, "Decision process for multiobjective, multi-item production-inventory system via interactive fuzzy satisficing technique," Computers \& Mathematics with Applications, vol. 49, no. 5-6, pp. 805-821, 2005.

[11] H.-C. Chang, J.-S. Yao, and L.-Y. Ouyang, "Fuzzy mixture inventory model involving fuzzy random variable lead time demand and fuzzy total demand," European Journal of Operational Research, vol. 169, no. 1, pp. 65-80, 2006.

[12] Z. Shao and X. Ji, "Fuzzy multi-product constraint newsboy problem," Applied Mathematics and Computation, vol. 180, no. 1, pp. 7-15, 2006.

[13] C.-M. Lee, "A Bayesian approach to determine the value of information in the newsboy problem," International Journal of Production Economics, vol. 112, no. 1, pp. 391-402, 2008.

[14] J. C. Hayya, T. P. Harrison, and D. C. Chatfield, "A solution for the intractable inventory model when both demand and lead time are stochastic," International Journal of Production Economics, vol. 122, no. 2, pp. 595-605, 2009.

[15] J. K. Dash, G. Panda, and S. Nanda, "Chance constrained programming problem under different fuzzy distributions," International Journal of Optimization. Theory Methods and Applications, vol. 1, no. 1, pp. 58-71, 2009.

[16] J.-S. Hu, H. Zheng, R.-Q. Xu, Y.-P. Ji, and C.-Y. Guo, "Supply chain coordination for fuzzy random newsboy problem with imperfect quality," International Journal of Approximate Reasoning, vol. 51, no. 7, pp. 771-784, 2010.

[17] G. Zhang, "The multi-product newsboy problem with supplier quantity discounts and a budget constraint," European Journal of Operational Research, vol. 206, no. 2, pp. 350-360, 2010.

[18] R.-H. Su, D.-Y. Yang, and W. L. Pearn, "Decision-making in a single-period inventory environment with fuzzy demand,"
Expert Systems with Applications, vol. 38, no. 3, pp. 1909-1916, 2011.

[19] K. A. M. Kotb and H. A. Fergany, "Multi-item EOQ model with both demand-dependent unit cost and varying leading time via geometric programming," Applied Mathematics, vol. 2, no. 5, pp. 551-555, 2011.

[20] O. Dey and D. Chakraborty, "A fuzzy random continuous review inventory system," International Journal of Production Economics, vol. 132, no. 1, pp. 101-106, 2011.

[21] R. Banerjee and S. Banerjee, "Solution of a probabilistic inventory model with chance constraints: a general fuzzy programming and intuitionistic fuzzy optimization approach," International Journal of Pure and Applied Sciences and Technology, vol. 9, no. 1, pp. 20-38, 2012.

[22] M. Nagare and P. Dutta, "On solving single-period inventory model under hybrid uncertainty," International Journal of Economics and Management Sciences, vol. 6, no. 4, pp. 290-295, 2012.

[23] M. A. Jahantigh, S. Khezerloo, A. A. Hosseinzadeh, and M. Khezerloo, "Solutions of fuzzy linear systems using ranking function," International Journal of Applied Operational Research, vol. 2, pp. 67-75, 2012.

[24] A. Karpagam and P. Sumathi, "New approach to solve fuzzy linear programming problems by the ranking function," Bonfring International Journal of Data Mining, vol. 4, no. 4, pp. 22-25, 2014.

[25] D. Dutta and P. Kumar, "Fuzzy inventory model without shortage using trapezoidal fuzzy number with sensitivity analysis," IOSR Journal of Mathematics, vol. 4, no. 3, pp. 32-37, 2012.

[26] S. Ding, "Uncertain multi-product newsboy problem with chance constraint," Applied Mathematics and Computation, vol. 223, pp. 139-146, 2013.

[27] H. Zolfagharinia and K. P. S. Isotupa, "Erratum on 'Optimal inventory control of empty containers in inland transportation system' Int. J. Prod. Econ. 133(1) (2011) 451-457," International Journal of Production Economics, vol. 141, no. 1, pp. 43-436, 2013.

[28] P. Majumder, U. K. Bera, and M. Maiti, "An EPQ model of deteriorating items under partial trade credit financing and demand declining market in crisp and fuzzy environment," Procedia Computer Science, vol. 45, pp. 780-789, 2015.

[29] S. M. Mousavi, J. Sadeghi, S. T. A. Niaki, N. Alikar, A. Bahreininejad, and H. S. C. Metselaar, "Two parameter-tuned metaheuristics for a discounted inventory control problem in a fuzzy environment," Information Sciences, vol. 276, pp. 42-62, 2014.

[30] H. N. Soni and M. Joshi, "A periodic review inventory model with controllable lead time and backorder rate in fuzzy-stochastic environment," Fuzzy Information and Engineering, vol. 7, no. 1, pp. 101-114, 2015.

[31] R. S. Kumar and A. Goswami, "A fuzzy random EPQ model for imperfect quality items with possibility and necessity constraints," Applied Soft Computing, vol. 34, pp. 838-850, 2015.

[32] N. Kazemi, E. Shekarian, L. E. C. Barrón, and E. U. Olugu, "Incorporating human learning into a fuzzy EOQ inventory model with backorders," Computers \& Industrial Engineering, vol. 87, pp. 540-542, 2015.

[33] J. K. Dash and A. Sahoo, "Optimal solution for a single period inventory model with fuzzy cost and demand as a fuzzy random variable," Journal of Intelligent \& Fuzzy Systems. Applications in Engineering and Technology, vol. 28, no. 3, pp. 1195-1203, 2015.

[34] M. Nagare, P. Dutta, and N. Cheikhrouhou, "Optimal ordering policy for newsvendor models with bidirectional changes in 
demand using expert judgment," OPSEARCH, vol. 53, no. 3, pp. 620-647, 2016.

[35] P. Raula, S. K. Indrajitsingha, P. N. Samanta, and U. K. Misra, "A Fuzzy Inventory Model for constant deteriorating item by using GMIR method in which inventory parameters treated as HFN," Open Journal of Applied and Theoretical Mathematics (OJATM), vol. 2, no. 1, pp. 13-20, 2016.

[36] I. Sangal, A. Agarwal, and S. Rani, "A fuzzy environment inventory model with partial backlogging under learning effect," International Journal of Computer Applications, vol. 137, no. 6 , pp. 25-32, 2016. 

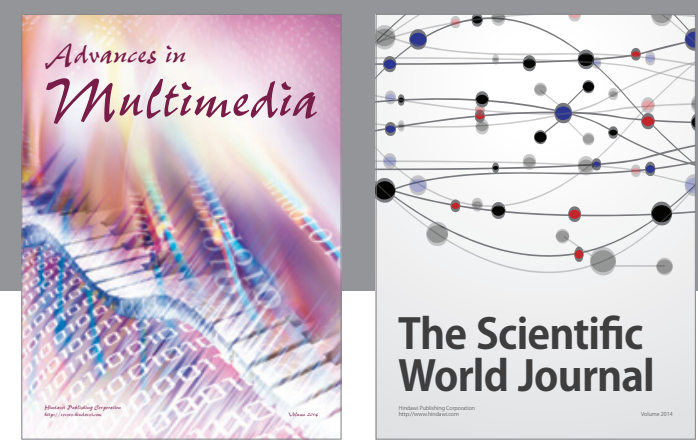

The Scientific World Journal
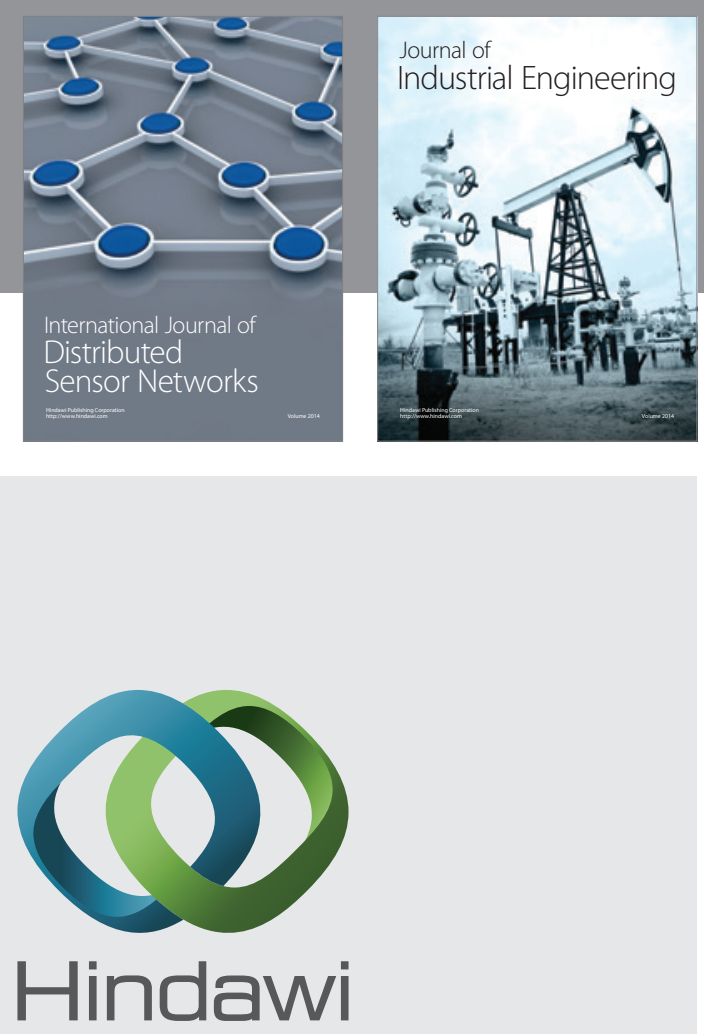

Submit your manuscripts at

http://www.hindawi.com

\section{Computer Networks} and Communications
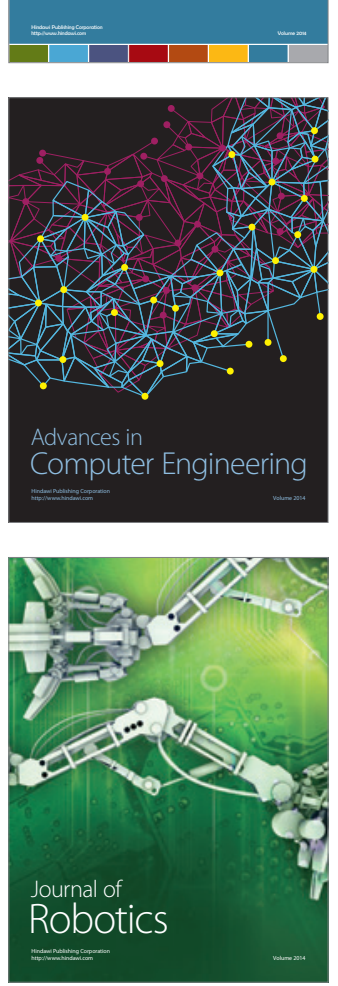
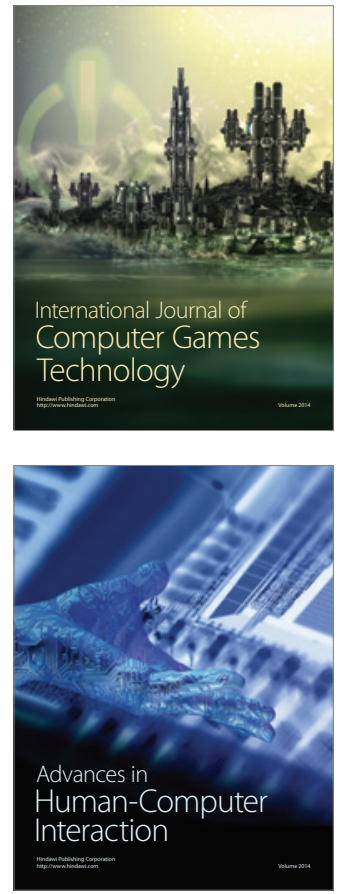
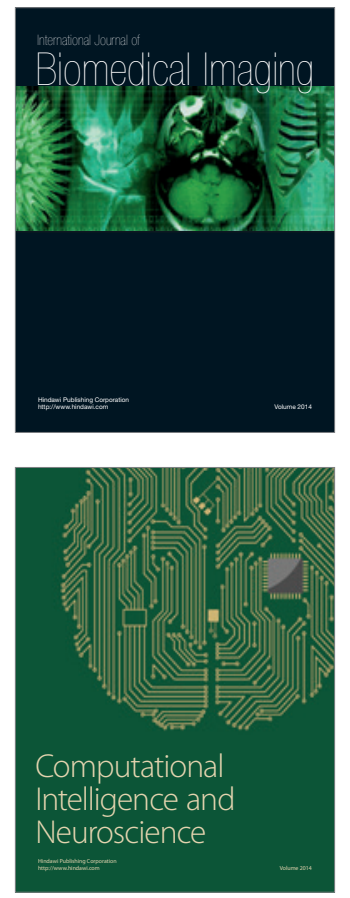
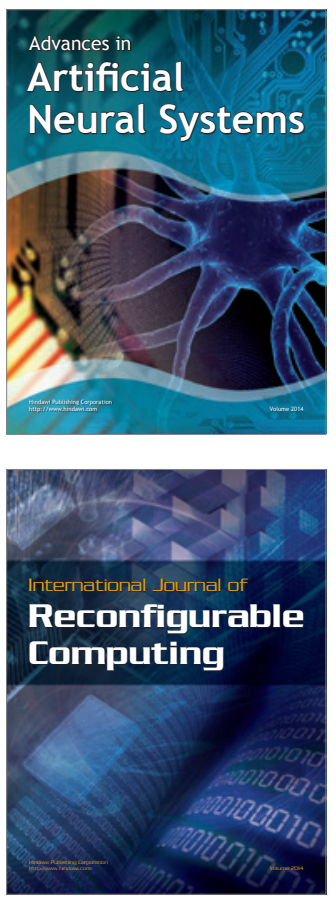
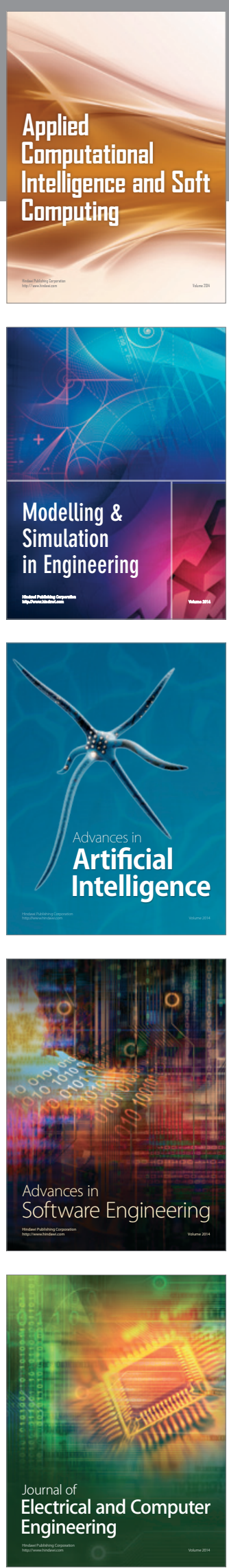